\title{
Venoactive Drugs, Summary of the Clinical Trials and Guidelines
}

\author{
Department of Surgery, Soonchunhyang University Seoul Hospital, Seoul, Korea
}

Sangchul Yun, M.D., Ph.D.

\begin{abstract}
The European Venous Forum guidelines recommend that venoactive drugs could be used as the primary treatment for symptoms and edema in all stages of chronic venous disease. In more advanced disease stages, venoactive drugs can be used in conjunction with surgery, sclerotherapy, thermal ablation, or compression therapy, and can improve the effectiveness of compression. It is usually not recommended to combine multiple venoactive drugs in the same prescription. Most venoactive drugs have common effects on swelling, pain, cramping, and weight. However, the clinical guidelines and the Cochrane review show that the effect is different for each drugs. Micronized purified flavonoid fractions might be more effective in venous ulcers, redness of the legs, and skin changes. Calcium dobesilate for restless legs syndrome, rutoside series for itching or abnormal sensations, and ruscus extract for fatigue are reportedly helpful in selecting appropriate venoactive drugs according to each symptom that patients complain of. In particular, the use of micronized purified flavonoid fraction seems to be possible for therapeutic purposes in patients with $\mathrm{CO}$ and $\mathrm{C} 1$ who exhibit temporary venous reflux, and patients with venous ulcers and post thrombotic syndrome. Therefore, it is necessary to use appropriate venoactive drugs according to the patient's symptoms and the severity of the disease. (Ann Phlebology 2021;19:21-26)
\end{abstract}

Key Words: Varicose vein, Chronic venous insufficiency, Venoactive drugs, Clinical practice guidelines, Cochrane review

\section{INTRODUCTION}

In Belgium and Luxembourg, a study of the patients with chronic venous insufficiency (CVI) found that the most common symptom was heaviness in the legs, which occurred

\footnotetext{
Corresponding author : Sangchul Yun, 59 Daesakwan-ro, Yongsan-gu, Seoul 04401, Korea, Department of Surgery, Soonchunhyang University Seoul Hospital Tel: 02-710-3240, Fax: 02-749-0449

E-mail: ys6325@schmc.ac.kr
}

in $70 \%$ of patients, about $50 \%$ experiencing pain and $20 \%$ complaining of itching or paresthesia. Seventy-five percent of patients reported experiencing more than one symptom (1). Several drugs are being used to compensate for compression therapy and lifestyle modification in the treatment of pain caused by CVI. Venoactive drugs (VAD) (flavonoids, saponins, calcium dobesilate, red vine leaf extract) and rheologic agents (aspirin, pentoxifylline) which affect venous wall tension and blood flow, are included (2). Various drugs have been developed and used in patients with CVI, but there are many questions about their effectiveness.

Most of VADs are natural flavonoids extracted from plants, and synthetic drugs with similar properties are also used Table 1. In the Anatomical Therapeutic Chemical (ATC) system, VADs are classified as vasoprotective agents (C05, vasoprotectives, $\mathrm{C} 05 \mathrm{C}$, capillary stabilizing agents) (ATC 2020) (3). Within this category, the active substances of the drugs are divided into several groups according to the organ or therapeutic effect, pharmacological and chemical properties in which the active substances act. Although the mechanism of action of VADs has not been well known scientifically despite numerous studies examining its pharmacological and clinical properties, it is believed that VADs are associated with better microcirculation through improved venous tone and decreased capillary permeability $(2,4)$.

Most studies on VADs were conducted in patients with chronic venous insufficiency and were shown to be effective in reducing discomfort and swelling in the lower extremities. (4). VADs have also been reported on their effects on clinical improvement of varicose veins, reduction of swelling and inflammation, improvement of microcirculation, and venous ulcer healing $(4,5)$. Therefore, the author intends to investigate the clinical usefulness of VADs through the trials and clinical guidelines reported so far. 


\section{COCHRANE REVIEWS 2020}

In the 2005 Cochrane Review, the efficacy of all VADs was analyzed and reported in 4413 patients included in 44 studies published up to 2005 (6). Although VADs showed some effects in reducing edema, etc., data were insufficient to evaluate the benefits to other signs and symptoms of venous insufficiency. There was also a lack of quantified data on quality of life. Subsequently, in 2020, the Cochrane group re-examined the data registered until November 12, 2019, and reported a second update on VADs (4). In a total of 56 studies, a systematic review of the literature including 7,690 patients was conducted, and various drugs were included in the analysis. Moderate-certainty evidence showed that most VADs could slightly reduce lower extremity edema (RR $0.70,95 \%$ CI 0.63 to $0.78 ; 13$ studies; 1245 participants) and reduced ankle circumference (mean difference $-4.27 \mathrm{~mm}, 95 \%$ CI -5.61 to $-2.93 \mathrm{~mm} ; 15$ studies; 2010 participants) compared to placebo. Moderate-certainty evidence showed that VADs showed little difference in quality of life compared to placebo (SMD - $0.06,95 \%$ CI -0.22 to 0.10 ; five studies; 1639 participants), Low-certainty evidence may have little effect on ulcer healing (RR 0.94, 95\% CI 0.79 to 1.13; six studies; 461 participants). Finally, 37 studies of side effects were reported. Consolidated data suggests that VADs may have slightly increased side effects compared to placebo (RR $1.14,95 \%$ CI 1.02 to $1.27 ; 37$ studies; 5789 participants; moderate-certainty evidence). The most frequently reported side effects were gastrointestinal disorders, but most were short-term data, and long-term data could not be evaluated.

Additionally, an analysis of the symptom effect of each drug type was conducted separately. Swelling, pain, and cramping were all improved in common, but for other symptoms, the effect was different for each drug. Unlike other medications, diosmin-based drugs could reduce heaviness (Standardized mean difference, SMD -0.69 [-1.02 to -0.36]) (4). Rutin, also called rutoside, quercetin-3-Orutinoside, and sophorin, is a glycoside that combines flavonol quercetin and disaccharide rutinose ( $\alpha$-L-rhamnopyranosyl$(1 \rightarrow 6)-\beta-D-$ glucopyranose). It is a flavonoid found in various plants including citrus spices. Rutin-based drugs are effective in swelling, pain, and cramping, and unlike other drugs, they are reported to be effective in itching (SMD $-0.58,-1.10$ to -0.06 ) and abnormal sensations (paresthesias, RR $0.55,0.37$ to 0.83 ) (4). Among the synthetic drugs, calcium dobesilate has a common effect on swelling, pain, and cramping, and unlike the others, calcium dobesilate can reduce restlessness in the legs (RR $0.73,0.59$ to 0.91 ).

Table 1. Summary of the category, names, brand names for each venoactive drug $(16,29)$

\begin{tabular}{|c|c|c|c|c|}
\hline Group & Substance & Origin & $\begin{array}{l}\text { Recommendation } \\
\text { grade } \\
\text { (2018 UIP } \\
\text { guideline) }\end{array}$ & Commercial products in Korea \\
\hline \multirow[t]{4}{*}{$\begin{array}{l}\text { Gamma-benzopyrones } \\
\text { (flavonoids) }\end{array}$} & Diosmin & Citrus spp. & 1 & $\begin{array}{c}\text { 조아디오스민(조아제약), 디오맥스(동아제약), } \\
\text { 베노론(한올바이오파마), 치센(동국제약) }\end{array}$ \\
\hline & MPFF* & Rutaceae aurantiae & 1 & $\begin{array}{l}\text { 베니톨(광동제약), 플라벤정(태극제약), } \\
\text { 프라린정(경남제약), 프라빈정(동성제약), } \\
\text { 치벤정(영일제약) }\end{array}$ \\
\hline & Rutin and rutosides & Sophora japonica & 1 & - \\
\hline & $\mathrm{HR}^{*}$ & Eucalyptus spp. & 1 & $\begin{array}{l}\text { 엘라스에이(조아제약), 케이나(삼진제약), } \\
\text { 헤모케어(오스틴제약) }\end{array}$ \\
\hline \multirow[t]{2}{*}{ Saponins } & Escin & Horse chestnut & 1 & - \\
\hline & Ruscus extract & Butcher's broom & 1 & - \\
\hline \multirow[t]{3}{*}{ Other plant extracts } & Anthocyans & Bilberry & $2 \mathrm{C}$ & 타겐에프(국제약품) \\
\hline & Proanthocyanidins & $\begin{array}{l}\text { Maritime pine, } \\
\text { Vitis vinifera }\end{array}$ & $2 \mathrm{C}$ & 엔테론(한림제약) \\
\hline & Centella asiatica & Centella, Gotukola & - & 센시아정(동국제약) \\
\hline \multirow[t]{2}{*}{ Synthetic product } & Calcium dobesilate & Synthetic & 2 & 독시움(일성신약) \\
\hline & Naftazon & Synthetic & - & 메디아벤(한국팜비오) \\
\hline
\end{tabular}

*MPFF: micronized purified flavonoid fraction, HR: 0-( $\beta$-Hydroxyethyl)-rutosides (troxerutin). 


\section{RECENT INTERESTING TRIALS FOR VADS}

Micronized purified flavonoid fraction (MPFF) is a mixed drug containing $90 \%$ and $10 \%$ diosmin and hesperidin, respectively. An interesting study of the effects of MPFF in 2017 was conducted in Russia. Doppler ultrasound was performed in the morning and evening in $96 \mathrm{C} 1$ patients, and vein size was measured. Transient venous reflux (TVR) was observed only in the evening in $55.2 \%$ of patients. MPFF (Daflon ${ }^{\circledR} 1000 \mathrm{mg}$; Servier, Suresnes, France) was prescribed for 90 days. In $92.5 \%$ of patients, TVR disappeared, the diameter of the great saphenous vein (5.69 $\mathrm{mm}$ to $5.14 \mathrm{~mm}, \mathrm{p}<0.01$ ) was significantly decreased, and the quality of life was also improved (QoL score 42.03 to $30.10, \mathrm{p}<0.01)(7)$. Through another systematic review of literature, it has been reported that the use of MPFF together with varicose vein surgery or sclerotherapy of the lower extremities helps improve clinical aspects such as VCSS and pain score $(8,9)$. In addition, the incidence of post-thrombotic syndrome (PTS) in patients with deep vein thrombosis significantly decreased after 12 months when the group treated with rivaroxaban and compression stockings was compared with the group treated with diosmin $(8.9 \%$ vs. $48.9 \%, \mathrm{p}<0.001)(10)$.

Centella asiatica was extracted from gotu grass. In a systematic review of the literature conducted in 2013, eight studies were summarized, and most showed improvement in venous reflux, clinical manifestations of venous insufficiency, venous wall tone, venous hypertension, and microcirculation. In these studies, the total triperpenic fraction of centella asiatica (TTFCA) was used at $60 \mathrm{mg}$ or $120 \mathrm{mg}$ per day, and the duration of use was 4 to 8 weeks (11). A study conducted in the UK reported that $120 \mathrm{mg} /$ day was more effective and safer than $60 \mathrm{mg} /$ day (12). A tablet of Censia sold as an over-the-counter drug on the market contains $30 \mathrm{mg}$ of Centella asiatica extract. It is recommended to take 1 2 Censia tablets per day, but it is less than the dose used in the previous trials.

\section{CLINICAL PRACTICE GUIDELINES}

The 2008 European venous forum (EVF) guidelines evaluated the efficacy and safety of VADs (13). The Cochrane Review and 2005 International Consensus Statement were reviewed and published on the efficacy of symptoms related to edema and chronic venous disease. It was recommended to use calcium dobesilate, MPFF, and hydroxyethyl-rutoside as Grade A, and to use VADs for the treatment of C6 lesions. Several studies have shown that MPFF is effective for venous ulcers. A meta-analysis of five trials administered MPFF as an adjuvant therapy with compressive stocking indicated that the use of MPFF was particularly significant for larger ulcers (relative risk rate= 40; 95\% CI: 6 to 87 , ulcers between 5 and $10 \mathrm{~cm}^{2}$ ) and long-lasting ulcers (relative risk rate $=44 ; 95 \% \mathrm{CI}$ : ulcers between 6 to 97 and 6 to 12 months), the conclusion that the time for venous ulcer healing was shortened was reported (14). Although not generally classified as VADs, Pentoxifylline was reviewed from 11 studies in a 2012 Cochrane review and saw that it could be an effective adjuvant to compression therapy to treat venous ulcers (relative risk $=2.2 ; 95 \%$ CI: 1.5 to 3.4 ). In particular, it was reported that it is effective even when used alone without compression treatment (relative risk=1.6; 95\% CI: 1.1 2.1) (15). For side effects, it was concluded that the safety of VADs was generally acceptable, except for liver toxicity of coumarin and benzarone. For other major types of VADs, the frequent side effects have been reported as gastrointestinal discomforts, skin rashes, headache, dizziness and insomnia.

The 2018 EVF guidelines' approach to determining the magnitude of the effect of individual VADs on specific symptoms provided evidence on the appropriate choice of VADs (16). The strengths of recommendations for VADs based on the clinical effectiveness and side effects of individual signs and symptoms are as follows: MPFF is strongly recommended to treat pain, heaviness, swelling, functional discomfort, convulsions, leg redness, skin changes, swelling, and quality of life (1, strong), with weak recommendation for paresthesia, and burning sensation (2, weak); ruscus +hesperidine methyl chalcone (HMC)+ascorbic acid is strongly recommended for treating pain, heaviness, swelling, leg fatigue, paresthesia, and swelling (1), with weak recommendations for convulsions and itching (2); for oxerutins, it had strong recommendations for the treatment of pain, heaviness, and spasms (1) and weak recommendations for edema (2); strong recommendations for treating pain, itching, and swelling using horse chestnut seed extract (HCSE) were reported (1); and in the case of calcium dobesilate, a weak recommendation (2) was given since it may induce agranulocytosis.

The 2008 ACCP guidelines recommend the addition of MPFF, rutosides, and sulodexide to compression therapy for patients with persistent venous ulcers (Grade 2, level of evidence B) (17). Patients with PTS are advised to avoid 
VADs (e.g., rutosides, defibrotide, hidrosmin) according to the 2012 ACCP guidelines [Grade 2, level of evidence C] (18). Two studies have been presented as references for this recommendation. In one study, the rutoside and control groups were compared in the treatment of ulcer-free PTS, and all patients wore compression stockings (19). Another study compared rutoside and hidrosmina (20). These two controlled studies found that rutosides may reduce ankle swelling, but not most PTS symptoms (RR 1.14, 95\% CI $0.85 \sim 1.52$ ). Because these results are inconsistent and inaccurate, the quality of the evidence is low. In addition, rutosides may be related to side effects (RR 2.04, 95\% CI $0.76 \sim 5.51$ ), most of which were symptoms related to gastrointestinal disorders.

\section{CLINICAL PRACTICE GUIDELINES (CPG) OF THE SOCIETY FOR VASCULAR SURGERY (SVS) AND THE AMERICAN VENOUS FORUM (AVF), $2011 \& 2014$}

In 2011, CPG of the SVS and the AVF recommended the use of VADs (diosmin, hesperidin, rutosides, sulodexide, MPFF or horse chestnut seed extract [aescin]) in patients with pain and swelling due to chronic venous disease [Grade 2, level of evidence B]. To accelerate the healing of venous ulcers, it is recommended to use pentoxifylline or MPFF with compression treatment where possible [Grade 2, level of evidence B] (21). In 2014, CPG of the SVS and the AVF recommended treatment with pentoxifylline or MPFF in combination with compression therapy for long-lasting or large venous leg ulcers [Grade 1, level of evidence B] (22).

European clinical practice guidelines (CPG of the ESVS, 2015) recommend that VADs be considered as a treatment option for swelling and pain caused by chronic venous disease [Class IIa, level of evidence A] (23). Sulodexide and MPFF were recommended to be considered as adjuvants to compression therapy in patients with venous ulcers [Class IIa, level of evidence A]. However, unlike previous studies or clinical practice guidelines, the basic use of zinc, oral antibiotics, HCSE and pentoxifylline in venous ulcers was not recommended [Class III, level of evidence B]. According to a recent review, a prospective randomized study reported that pentoxifylline and MPFF as well as other flavonoid derivatives had clinical effects in patients with grade C 4-6 CVD (24). A review of the Cochrane database shows that pentoxifylline is effective as an adjuvant therapy for compression, but it is also effective in the absence of compression (15). Other studies have shown that the ulcer healing time is accelerated when patients are treated with pentoxifylline $(25,26)$. In a recent systematic review, the authors recommended the use of pentoxifylline for the treatment of venous ulcers (5). Based on this evidence, pentoxifylline seems to be effective for venous ulcers, and it is not clearly explained why the use of pentoxifylline is not recommended by the ESVS guidelines.

In 2016, the Deutsche Dermatologische Gesellschaft specifically recommended the duration of use of VADs; when evaluating the clinical effect in daily life, $2 \sim 4$ weeks of continuous intake is necessary to fully exert their effect [Grade 1-C] (27). However, patients ask if these drugs can be taken for a long time, and long-term use containing high doses of polyphenols may cause dysbiosis. The answers to these concerns were revealed in the following study. A meta-analysis of polyphenol supplementation was reported in the United States in 2020. Significant heterogeneity analyses have shown that polyphenol supplements benefit human health by forming species of microbes in the gut (28).

\section{CONCLUSION}

TThe EVF guidelines recommended the following regarding the use of VADs. These drugs can be used as the primary treatment for symptoms and edema in all stages of chronic venous disease. In more advanced disease stages, VADs can be used in conjunction with stripping, endovenous thermal ablation, sclerotherapy, or compressive stocking, and can improve the effectiveness of compression. (13). Most VADs showed a common effect on swelling, pain, cramping, and heaviness. However, as analyzed and reported by the Cochrane Review in 2020 and the EVF Guidelines in 2018, VADs seem to differ in efficacy by ingredient, and improved clinical patterns were also varied $(4,16)$. Therefore, it is necessary to use appropriate VADs according to the severity of the patient's symptoms and stage of disease.

\section{ACKNOWLEDGEMENTS}

The same topic was presented at the 40th Conference of the Korean Society for Phlebology on April 18, 2021. 


\section{CONFLICT OF INTEREST}

The author declares no potential conflict of interest.

\section{REFERENCES}

1) Vuylsteke ME, Colman R, Thomis S, Guillaume G, Degrande $\mathrm{E}$, Staelens I. The influence of age and gender on venous symptomatology. An epidemiological survey in Belgium and Luxembourg. Phlebology. 2016;31:325-33.

2) Roh YN. The Mechanism and Clinical Evidence of Venoactive Drugs. Annals of Phlebology. 2019;17:18-22.

3) Anatomical Therapeutic Chemical (ATC) system classification.

4) Martinez-Zapata MJ, Vernooij RW, Simancas-Racines D, Uriona Tuma SM, Stein AT, Moreno Carriles RMM, et al. Phlebotonics for venous insufficiency. Cochrane Database Syst Rev. 2020;11:Cd003229.

5) Kitchens BP, Snyder RJ, Cuffy CA. A Literature Review of Pharmacological Agents to Improve Venous Leg Ulcer Healing. Wounds. 2020;32:195-207.

6) Martinez MJ, Bonfill X, Moreno RM, Vargas E, Capellà D. Phlebotonics for venous insufficiency. Cochrane Database Syst Rev. 2005:Cd003229.

7) Tsukanov YT, Nikolaichuk AI. Orthostatic-loading-induced transient venous refluxes (day orthostatic loading test), and remedial effect of micronized purified flavonoid fraction in patients with telangiectasia and reticular vein. Int Angiol. 2017;36:189-96

8) Mansilha A, Sousa J. Benefits of venoactive drug therapy in surgical or endovenous treatment for varicose veins: a systematic review. Int Angiol. 2019;38:291-8.

9) Bogachev VY, Boldin BV, Turkin PY. Administration of Micronized Purified Flavonoid Fraction During Sclerotherapy of Reticular Veins and Telangiectasias: Results of the National, Multicenter, Observational Program VEIN ACT PROLONGED-C1. Adv Ther. 2018;35:1001-8.

10) Schastlivtsev I, Lobastov K, Barinov V, Kanzafarova I. Diosmin 600 in adjunction to rivaroxaban reduces the risk of post-thrombotic syndrome after femoropopliteal deep vein thrombosis: results of the RIDILOTT DVT study. Int Angiol. 2020;39:361-71.

11) Chong NJ, Aziz Z. A Systematic Review of the Efficacy of Centella asiatica for Improvement of the Signs and Symptoms of Chronic Venous Insufficiency. Evid Based Complement Alternat Med. 2013;2013:627182.

12) Incandela L, Belcaro G, De Sanctis MT, Cesarone MR, Griffin M, Ippolito E, et al. Total triterpenic fraction of Centella asiatica in the treatment of venous hypertension: a clinical, prospective, randomized trial using a combined microcirculatory model. Angiology. 2001;52 Suppl 2:S61-7.
13) Nicolaides AN, Allegra C, Bergan J, Bradbury A, Cairols $\mathrm{M}$, Carpentier $\mathrm{P}$, et al. Management of chronic venous disorders of the lower limbs: guidelines according to scientific evidence. Int Angiol. 2008;27:1-59.

14) Coleridge-Smith P, Lok C, Ramelet AA. Venous leg ulcer: a meta-analysis of adjunctive therapy with micronized purified flavonoid fraction. Eur J Vasc Endovasc Surg. 2005;30:198-208.

15) Jull AB, Arroll B, Parag V, Waters J. Pentoxifylline for treating venous leg ulcers. Cochrane Database Syst Rev. 2012;12:Cd001733.

16) Nicolaides A, Kakkos S, Baekgaard N, Comerota A, de Maeseneer M, Eklof B, et al. Management of chronic venous disorders of the lower limbs. Guidelines According to Scientific Evidence. Part I. Int Angiol. 2018;37:181-254.

17) Kearon C, Kahn SR, Agnelli G, Goldhaber S, Raskob GE, Comerota AJ. Antithrombotic therapy for venous thromboembolic disease: American College of Chest Physicians Evidence-Based Clinical Practice Guidelines (8th Edition). Chest. 2008;133(6 Suppl):454s-545s.

18) Kearon C, Akl EA, Comerota AJ, Prandoni P, Bounameaux $\mathrm{H}$, Goldhaber SZ, et al. Antithrombotic therapy for VTE disease: Antithrombotic Therapy and Prevention of Thrombosis, 9th ed: American College of Chest Physicians Evidence-Based Clinical Practice Guidelines. Chest. 2012;141 (2 Suppl):e419S-e496S.

19) Frulla M, Marchiori A, Sartor D, Mosena L, Tormene D, Concolato A, et al. Elastic stockings, hydroxyethylrutosides or both for the treatment of post-thrombotic syndrome. Thromb Haemost. 2005;93:183-5.

20) Monreal M, Callejas JM, Martorell A, Lisbona C, Lerma R. A Prospective Study of the Long-Term Efficacy of Two Different Venoactive Drugs in Patients with Post-Thrombotic Syndrome. Phlebology. 1994;9:37-40.

21) Gloviczki P, Comerota AJ, Dalsing MC, Eklof BG, Gillespie DL, Gloviczki ML, et al. The care of patients with varicose veins and associated chronic venous diseases: clinical practice guidelines of the Society for Vascular Surgery and the American Venous Forum. J Vasc Surg. 2011;53(5 Suppl):2s-48s.

22) O’Donnell TF, Jr., Passman MA, Marston WA, Ennis WJ, Dalsing M, Kistner RL, et al. Management of venous leg ulcers: clinical practice guidelines of the Society for Vascular Surgery ${ }^{\circledR}$ and the American Venous Forum. J Vasc Surg. 2014;60(2 Suppl):3s-59s.

23) Wittens C, Davies AH, Bækgaard N, Broholm R, Cavezzi A, Chastanet S, et al. Editor's Choice - Management of Chronic Venous Disease: Clinical Practice Guidelines of the European Society for Vascular Surgery (ESVS). Eur J Vasc Endovasc Surg. 2015;49:678-737.

24) Gohel MS, Davies AH. Pharmacological agents in the treatment of venous disease: an update of the available 
evidence. Curr Vasc Pharmacol. 2009;7:303-8.

25) Dale JJ, Ruckley CV, Harper DR, Gibson B, Nelson EA, Prescott RJ. Randomised, double blind placebo controlled trial of pentoxifylline in the treatment of venous leg ulcers. Bmj. 1999;319:875-8.

26) Falanga V, Fujitani RM, Diaz C, Hunter G, Jorizzo J, Lawrence PF, et al. Systemic treatment of venous leg ulcers with high doses of pentoxifylline: efficacy in a randomized, placebo-controlled trial. Wound Repair Regen. 1999;7:208-13.
27) Stücker M, Debus ES, Hoffmann J, Jünger M, Kröger K, Mumme A, et al. Consensus statement on the symptombased treatment of chronic venous diseases. J Dtsch Dermatol Ges. 2016;14:575-83.

28) Ma G, Chen Y. Polyphenol supplementation benefits human health via gut microbiota: A systematic review via metaanalysis. Journal of Functional Foods. 2020;66:103829.

29) Pitsch F. Recent Guidelines in Chronic Venous Disease: the place of Daflon $500 \mathrm{mg}$. Phlebolymphology. 2011;18: 24-9. 